\title{
Astigmatism of the Ex Vivo Human Lens: Surface and Gradient Refractive Index Age-Dependent Contributions
}

\author{
Judith Birkenfeld, Alberto de Castro, and Susana Marcos \\ Instituto de Óptica "Daza de Valdés," Consejo Superior de Investigaciones Científicas (CSIC), Madrid, Spain
}

Correspondence: Susana Marcos, Serrano, 121, 28006 Madrid, Spain; susana@io.cfmac.csic.es.

Submitted: January 17, 2015

Accepted: June 1, 2015

Citation: Birkenfeld J, de Castro A, Marcos S. Astigmatism of the ex vivo human lens: surface and gradient refractive index age-dependent contributions. Invest Ophthalmol Vis Sci. 2015;56:5067-5073. DOI:10.1167/ iovs.15-16484
Purpose. We estimated the contribution of the gradient refractive index (GRIN) and lens surfaces to lens astigmatism and lens astigmatic angle as a function of age in human donor lenses.

Methods. Human lenses were imaged, ex vivo, with 3D-spectral optical coherence tomography (OCT) and their back focal length was measured using laser ray tracing. The contribution of lens surfaces and GRIN to lens astigmatism were evaluated by computational ray tracing on the GRIN lens and a homogenous equivalent index lens. Astigmatism magnitude and relative astigmatic angle of and between lens surfaces, GRIN lens, and lens with homogeneous refractive index were evaluated, and all results were correlated with age.

REsults. The magnitude of astigmatism in the anterior lens surface decreased with age (slope $=$ -0.005 diopters $[\mathrm{D}] / \mathrm{y} ; r=0.397, P=0.018$ ). Posterior surface astigmatism and lens astigmatism were not age-dependent. Presence of GRIN did not alter significantly the magnitude or axis of the lens astigmatism. The astigmatism of GRIN lens and lens with homogeneous refractive index correlated with anterior lens surface astigmatism (GRIN, $P=3.9 \mathrm{E}-6, r=$ 0.693 ; equivalent refractive index lens, $P=4.1 \mathrm{E}-4, r=0.565)$. The astigmatic angle of posterior surface, GRIN lens, and homogeneous refractive index lens did not change significantly with age.

Conclusions. The axis of lens astigmatism is close to the astigmatic axis of the anterior lens surface. Age-related changes in lens astigmatism appear to be related to changes in the anterior lens astigmatism. The influence of the GRIN on lens astigmatism and the astigmatic axis is minor.

Keywords: crystalline lens, GRIN, astigmatism
$T^{\mathrm{h}}$ The human crystalline lens is an optical element that, 1 together with the cornea, refracts and transmits the light to form an image on the retina. Its optical properties are dependent on its shape and its index of refraction, which is not homogeneous, and is known to peak in the center of the lens and decrease toward the cortex in all directions. The gradient refractive index (GRIN) ${ }^{1-6}$ and lens shape $e^{7-11}$ change with age, resulting in an age-dependency of the optical properties of the lens and, therefore, the whole eye. It has been shown that the lens spherical aberration shifts toward more positive values with age. , 10,12-14 $^{2}$ Measurements of total and corneal spherical aberrations have shown that the crystalline lens is primarily responsible for age-related loss of corneal/internal balance of spherical aberration. ${ }^{14-16}$

Several studies have reported changes in the geometrical shape of the lens with age, such as lens thickening and lens surface steepening. ${ }^{1,8,11,12,17-19}$ Since the eye does not tend to become myopic with age, it was postulated that the lens GRIN changed in such a way that it compensated for lens surface steepening to maintain lens power constant with age (so-called lens paradox). ${ }^{1,11,20}$

Direct measurements of the GRIN distribution in the lens and its change with age are scarce. Earlier studies used a reflectometric fiberoptic sensor, ${ }^{21}$ or magnetic resonance imaging. ${ }^{22,23}$ More recently, a technique based on optical coherence tomography (OCT) has been proposed, ${ }^{5}$ which has allowed estimates of the GRIN distribution ex vivo in twodimension (2D) in human donor lenses as a function of age, ${ }^{24}$ and cynomolgus monkey lenses as a function of accommodation, ${ }^{25}$ and in three-dimensional (3D) ex vivo in porcine lenses and human lenses of different ages. ${ }^{6,26}$ In humans, it has been found that, with age, the GRIN profile changes from a parabolic shape to a central plateau with constant index and a rapid decrease toward the periphery, ${ }^{1,22,24}$ more prominent in the meridional than in the axial direction. ${ }^{26}$ Ray tracing estimates of the spherical aberration in ex vivo human lenses using the measured geometrical shape and the estimated GRIN revealed a significant contribution of the GRIN in the negative spherical aberration of young lenses, ${ }^{27}$ as well as a shift of the spherical aberration toward less negative values with aging. ${ }^{26}$ There was an excellent agreement between the estimated spherical aberration and that measured by laser ray tracing (LRT) in the same lenses. ${ }^{28}$ Previous studies ${ }^{29,30}$ also evaluated the impact of the GRIN, as opposed to the assumed constant index, in the quantification of the posterior lens shape from OCT imaging.

While the contributions of the cornea and lens, as well as the lens shape and GRIN to spherical aberration have been relatively well studied, to our knowledge a similar analysis has not been performed on the relative contributions to astigmatism, likely because most lens studies to date only had access to 2D data. 
Compensatory effects of anterior and posterior corneal astigmatism have been reported. ${ }^{31-37}$ Also, longitudinal studies report changes in the cornea from with-the-rule (direct) to against-the-rule (indirect) astigmatism with age. ${ }^{38}$ Measurements of total and corneal astigmatism from ocular refraction $^{39,40}$ or ocular aberration measurements ${ }^{15,41}$ suggest at least partial compensation of total and internal astigmatism. In those studies, the contributions of the posterior cornea and lens to the internal optics cannot be isolated. In an interesting study using an ophthalmophakometric technique, Elawad et al. ${ }^{42}$ estimated the ocular component contributions to residual astigmatism in human eyes, and found that, while the astigmatic contributions of the posterior corneal and lens surfaces were predominantly inverse, direct astigmatism came from the anterior lens surface. Similar conclusions were reached by Dunne et al. ${ }^{43}$ in a later work, although they recognized that the method was indirect and prone to accumulated experimental errors.

Lens surface astigmatism was studied in human cadaver lenses using a corneal topography system. ${ }^{44}$ In this study, radius of curvature and shape factor were fitted over 18 meridians. In most cases the variations were random, but in some cases the variations indicated the presence of regular lens astigmatism.

To our knowledge, the only direct measurement of crystalline lens surface astigmatism on ex vivo human lenses comes from surface topographic analysis (using OCT). ${ }^{45}$ In that study it was found that astigmatism was the predominant lens surface aberration. A significant change in the amount of surface astigmatism aberration with age was not found, although the relative angle of astigmatism between the anterior and posterior lens surfaces tended to decrease with age, indicating a potential decrease in the compensatory effects of anterior and posterior lens astigmatism with age. The study did not consider potential effects of the GRIN distribution with age.

Access to lens shape and GRIN in 3D opens the possibility of evaluating the relative role of lens surfaces and GRIN astigmatism to lens astigmatism, and the potential changes with age, as in similar analysis of contributors of spherical aberration.

For this study, we used 3D spectral OCT data on 35 isolated human lenses $(47.6 \pm 13.4$ years), of which shape and GRIN had been characterized in 3D. ${ }^{26}$ We present here lens surface astigmatism, and lens astigmatism, and their changes in magnitude and axis with age. In particular, the contribution of surface and GRIN astigmatism to the lens astigmatism was studied assuming an equivalent refractive index, and the estimated GRIN.

It is particularly interesting to understand the contribution of the different lens surfaces to the optical quality of the eye in the context of potential replacements of the presbyopic or cataractous crystalline lens. This contribution seems especially relevant for the management of astigmatism with toric intraocular lenses, since it explains the contribution of the eventually replaced crystalline lens to the astigmatism of the eye.

\section{Methods}

\section{Human Lens Samples and Preparation}

All human donor eyes were received from the Transplant Service Foundation (TSF) Eye Bank in Barcelona, Spain. During the transportation, eyes were packed individually in sealed vials at $4^{\circ} \mathrm{C}$, wrapped in gauze soaked in a preservation medium (Dulbecco's modified Eagle's medium [DMEM]/F-12, HEPES, no phenol red; GIBCO, Carlsbad, CA, USA). Presence of any form of cataract was considered an exclusion criterion for the study. Before shipment the corneas had been removed (for corneal transplant purposes) and in some cases sections of the sclera. However, the vitreous and the choroid were preserved and provided a safe transportation environment for the lens. All lenses arrived 1 to 2 days post mortem, and were measured within 24 hours.

A total of 35 eyes from 30 human donors were used in the study. Ages ranged between 19 and 71 years. Before the experiment, the lens zonules were carefully cut and the lens was extracted from the eye with soft tweezers and handled mainly using the remaining zonules rather than touching the lens capsule. After extraction the lens was immediately immersed in DMEM at room temperature. During the measurements, the lens was placed on a ring in a DMEM-filled cuvette. The whole measurement took up to 2 hours. Swollen or damaged lenses were identified with the OCT images and excluded from the study.

Handling and experimental protocols had been approved previously by the Institutional Review Boards of TSF and CSIC. Methods for securing human tissue were in compliance with the Declaration of Helsinki.

\section{OCT Imaging}

The cuvette containing the lens was placed on a horizontal platform, and imaged using a custom developed high resolution spectral OCT system described in detail elsewhere. ${ }^{46} \mathrm{~A}$ mirror system above the platform assured that the OCT beam was directed toward the upper lens surface. The system uses an 840-nm superluminescent (SLD) diode as illumination source and obtained 3D images composed of 1668 A-Scans, and 70 B-Scans on a $12 \times 12-\mathrm{mm}$ lateral area. The acquisition time was 4.5 seconds, and the axial resolution was calculated to $6.9 \mu \mathrm{m}$ in tissue. The accuracy of the external surfaces of the lens could be limited by the axial resolution. However, conservative calculations showed little impact on the reported astigmatism.

The lens axis could be easily centered with the OCT set to visualization mode, which displays two orthogonal B-scans in an interval of 0.5 seconds. The manually adjustable platform on which the cuvette was set up was aligned until a specular reflection was seen from the surfaces of the lens.

All lenses were imaged in two different focal planes, to allow visualization of lens surfaces and the cuvette holding the lens. The images were merged into one complete 3D image (anterior surface, posterior surface, and cuvette surface) using a custom-developed merging algorithm. ${ }^{26}$ The lens was first completely imaged with the anterior surface facing the OCT beam, and then was flipped around a predetermined axis and imaged again with the posterior surface up. The aberration present on the image of the cuvette, which should be the same for both orientations of the lens, was used to correct possible alignment errors.

\section{Laser Ray Tracing}

After the OCT measurements, the focal length of each lens was measured with a custom developed LRT. The system combines a 2-mirror galvanometric scanning system with a $400-\mathrm{mm}$ collimating lens. The illumination source is a superluminescent diode $(849 \mathrm{~nm})$. The system and its calibration have been described in previous publications. ${ }^{6,26}$ In brief, the crystalline lenses were placed horizontally in a cuvette (anterior up position), positioned on a stable platform. A motorized CMOS camera was placed right under the cuvette (whose base was optical quality glass) to capture a series of through-focus images from the beam directed through the lens. The lenses 
were aligned with the LRT, such that the lens and the principal ray were collinear to the center of the CMOS camera, in the entire focus range $(35 \mathrm{~mm}$, which was the axial range of the motor). The focal lengths of each lens were measured projecting rings of light of different diameters ( 2 and $4 \mathrm{~mm}$ ) onto the crystalline lens' upper surface. The refraction through the glass was compensated using a calibration method that involved measurements with a set of artificial lenses ${ }^{48}$ (Edmund Optics, Inc., Barrington, NJ, USA). The estimated precision of the focal length measurements was $0.8 \mathrm{~mm}$. The focal length data were used as input data for the GRIN reconstruction algorithm as described by Birkenfeld et al. ${ }^{26}$

\section{Image Processing and GRIN Reconstruction}

All OCT images were corrected for optical distortion and fan distortion, and all surfaces (lens and cuvette) were fitted with Zernike polynomials (up to seventh order) within a 6-mm pupil. As described in detail in previous references, ${ }^{5,26}$ the method involves acquiring images of the crystalline lens in two different orientations, with the anterior surface up and with the posterior surface up. The aberration of the cuvette was used to compensate for potential alignment errors while flipping. ${ }^{5}$ Following correction of refraction by the preservation medium, the method allowed reconstruction of the undistorted lens shape. ${ }^{5}$ Furthermore, the posterior surface of the lens is distorted by the anterior surface and GRIN.

The GRIN was reconstructed by means of a search algorithm using the optical path measured from the OCT images and the measured back focal length. ${ }^{6}$ The gradient refractive index is described as a 4-variable model expressed in polar coordinates with the origin in the center of the GRIN as

$$
n(\rho, \theta)=n_{N}-\Delta n \cdot\left(\frac{\rho}{\rho_{S}}\right)^{p(\theta)}
$$

where $n_{N}$ is the refractive index of the nucleus, $\Delta n$ the difference between the refractive index of surface and nucleus, $\rho_{S}$ is the distance between nucleus and surface, and $p(\theta)$ is the exponential decay for axial $(p 1)$ and meridional $(p 2)$ direction. The axial decay $p 1$ is constant across meridians, while $p 2$ can vary to account for differences between meridians. This model was fully described in previous publications. ${ }^{5,6,26}$

The change of the power exponent $p 2$, that is, the decay of the GRIN in the meridional direction, is an indicator of the GRIN contribution to astigmatism. A constant $p 2$ across the lens is indicative of no GRIN astigmatism. A sinusoidal variation of $p 2$ across lens meridians is indicative of the presence of GRIN astigmatism. In all computations the center of the GRIN is assumed to be placed at a distance from the anterior vertex equal to 0.41 times the central thickness of the lens. ${ }^{47}$

\section{Calculation of Lens Astigmatism (Magnitude and Axis)}

Lens GRIN Astigmatism. The lens astigmatism was calculated using the measured lens shape and the reconstructed GRIN by means of a computational ray tracing analysis. ${ }^{5,6}$ The calculated wave aberrations were fit by Zernike polynomials. The magnitude of astigmatism $C$ and angle $\alpha$ were calculated $^{48}$ as

$$
\begin{aligned}
& C=-2^{*} \sqrt{\mathrm{J}_{45}^{2}+\mathrm{J}_{180}^{2}} \\
& \alpha=\tan ^{-1}\left(\frac{J_{45}}{J_{180}}\right) / 2,
\end{aligned}
$$

where $J_{180}$ is the power at axis $\alpha=180^{\circ}$ and $J_{45}$ is the power at axis $\alpha=45^{\circ} ; J_{180}$ and $J_{45}$ are related with the Zernike coefficients by the following expressions ${ }^{49}$ :

$$
\begin{aligned}
& J_{180}=\left(-2 \sqrt{6 /} r^{2}\right)\left(Z_{2}^{2}\right) \\
& J_{45}=\left(-2 \sqrt{6 /} r^{2}\right)\left(Z_{2}^{-2}\right)
\end{aligned}
$$

where $Z_{2}{ }^{-2}$ and $Z_{2}{ }^{2}$ are the corresponding astigmatism Zernike terms, and $r$ is the pupil radius ( $3 \mathrm{~mm}$ in this study).

Equivalent Refractive Index Lens Astigmatism. Lens astigmatism was calculated for all lenses considering a GRIN and a homogeneous equivalent refractive index. The equivalent refractive index is defined as the homogeneous refractive index with the same measured geometry and focal length as the GRIN lens.

By comparing the lens astigmatism with GRIN and with a homogeneous refractive index we can assess the contribution of GRIN to the lens astigmatism.

Virtual ray tracing was performed, to obtain $Z_{2}^{-2}$ and $Z_{2}^{2}$. From the Zernike fittings, $C, J_{180}, J_{45}$, and the astigmatic axis were calculated using Equations 2 through 5 .

Surface Lens Astigmatism. The lens surface astigmatism $C_{S}$ was calculated as

$$
C_{S}=\left(n_{2}-n_{1}\right)\left(\frac{1}{R_{x}}-\frac{1}{R_{y}}\right),
$$

where $R_{x}$ and $R_{y}$ are the radii of curvature on the principal axis, and $n_{1}$ and $n_{2}$ are the refractive indices of the lens immersion medium and the lens cortex, respectively. The index of refraction of the lens cortex $\left(n_{2}\right)$ was taken directly from our GRIN reconstruction, individually for every lens. The refractive index of aqueous $\left(n_{1}\right)$ was assumed to be $1.336 .{ }^{50}$

Relative Astigmatic Angle. Since the orientation of the isolated lens during the measurements is not corresponding to its actual orientation in vivo (up, down, nasal, temporal), the calculated axis of astigmatism of the lens surfaces is arbitrary. However, the relative angle between the different axes of astigmatism (anterior, posterior, GRIN lens, lens with homogeneous refractive index) can be computed, and defined, in a range between $0^{\circ}$ and $90^{\circ}$.

Power Vector Analysis. To illustrate the magnitude of astigmatism and angle, the results are presented using a power vector graph. ${ }^{48}$ As indicated above, we assumed that the axis of astigmatism of the anterior lens surface is vertically aligned in all lenses. We investigated potential rotations between the astigmatic axis of posterior lens surface, GRIN lens, and lens with equivalent refractive index with respect to the vertical aligned anterior surface astigmatic axis, and potential agerelated changes.

\section{RESULTS}

\section{Change of Magnitude of Astigmatism With Age}

Figure 1 shows the change of the anterior and posterior lens surface astigmatism with age, fitted by linear regression. The anterior lens surface astigmatism decreases significantly with age $(r=0.397, P=0.018)$. The posterior lens surface astigmatism does not change significantly with age $(r=$ $0.189, P=0.276$ ).

Figure 2 shows the change of the lens magnitude of astigmatism with age for the crystalline lenses with the reconstructed GRIN $(r=0.359, P=0.034)$ in comparison with the lens magnitude of astigmatism in the same lenses assuming a homogeneous equivalent refractive index $(r=$ 


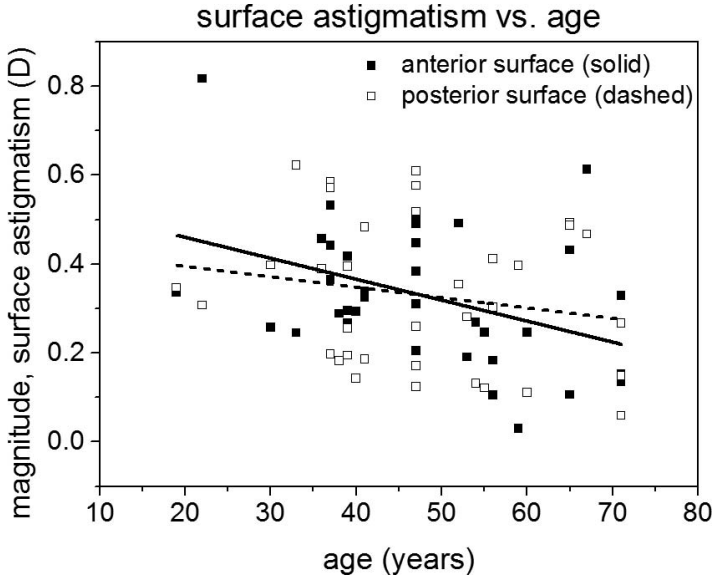

Figure 1. Change in the lens surfaces astigmatism with age (anterior surface, slope $=-0.005 \mathrm{D} / \mathrm{y}, r=0.397, P=0.018$; posterior surface, slope $=-0.002 \mathrm{D} / \mathrm{y}, r=0.189, P=0.276)$.

$0.380, P=0.024)$. The mean value of the magnitude of astigmatism was 1.46 diopters (D) for the GRIN lens and 1.02 $\mathrm{D}$ for the homogeneous refractive index lens.

Figure 3 shows a high correlation between the anterior lens surface astigmatism, and the GRIN lens astigmatism and the homogeneous refractive index lens astigmatism (GRIN lens, $r=$ $0.693, P=3.9 \mathrm{E}-6$; equivalent refractive index lens, $r=0.565$, $P=4.1 \mathrm{E}-4)$. The posterior lens surface astigmatism did not correlate with the lens astigmatism (not shown). The mean astigmatism of anterior lens surface and posterior lens surface were $0.43 \pm 0.22$ and $0.41 \pm 0.19 \mathrm{D}$, respectively.

The GRIN lens astigmatism and the astigmatism of the lens with a homogeneous refractive index are similar and correlate well with each other ( $r=0.555, P<0.01$, not shown). The average astigmatism of the GRIN and equivalent refractive index lens were $1.22 \pm 0.82$ and $1.04 \pm 0.65 \mathrm{D}$, respectively.

\section{Relative Astigmatic Angles}

As the absolute orientation of the lens is not known, the analysis of the astigmatic axis can only be made in relative terms. In particular, we studied potential changes with age of the astigmatic angles between anterior and posterior surface and the difference in astigmatic axis between the GRIN lens and the lens with homogeneous refractive index.

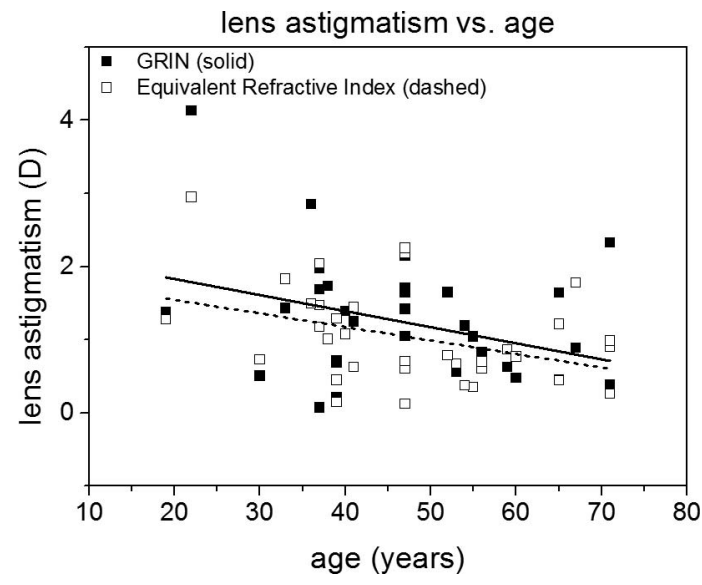

FIGURE 2. Change in the lens astigmatism with age (lens with GRIN, slope $=-0.022 \mathrm{D} / \mathrm{y}, r=0.359, P=0.034$; lens with equivalent refractive index, slope $=-0.018 \mathrm{D} / \mathrm{y}, r=0.380, P=0.024)$.

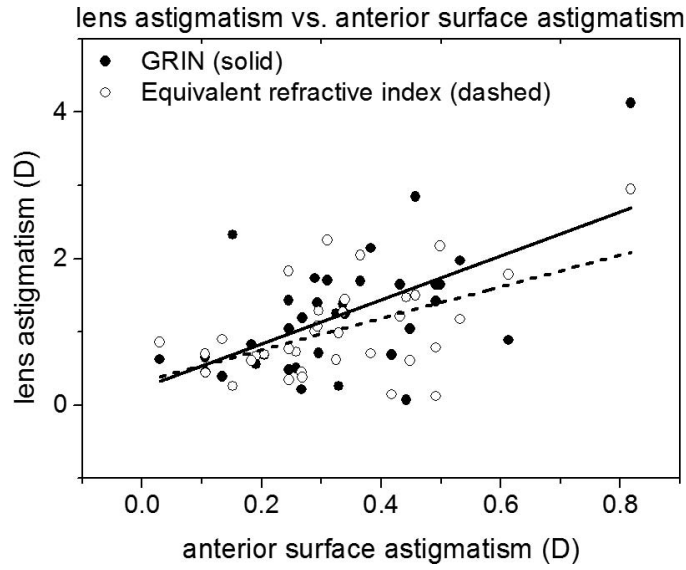

Figure 3. Correlation between the GRIN lens astigmatism and the anterior lens surface astigmatism $(r=0.693, P=3.9 \mathrm{E}-6)$ and the equivalent refractive index lens astigmatism and the anterior lens surface astigmatism $(r=0.565, P=4.1 \mathrm{E}-4)$

Figure 4 shows polar plots (using the power vector analysis notation) of astigmatic magnitude and axis of the lens surfaces, the GRIN lens, and the lens with homogeneous refractive index. The angle shown for the posterior lens surface, GRIN lens, and homogeneous refractive index lens are expressed relative to the anterior lens surface. Each dot in the plot represents one lens.

The average relative angle between anterior and posterior lens surface was $28.5^{\circ}$. In $68.57 \%$ of the lenses the relative angle was $<45^{\circ}$.

The axis of the GRIN lens was almost aligned with the axis of the anterior lens surface. The average relative angle between anterior surface axis and GRIN axis was $8.9^{\circ}$. In $88.57 \%$ of the lenses the relative angle was $<45^{\circ}$ (with $65.71 \%$ of all lenses having a relative angle $<15^{\circ}$ ).

The presence of GRIN did not seem to have a large influence on the astigmatic axis. The relative angle between the astigmatic axis of the GRIN lens and the lens with homogeneous refractive index lens was on average $15.5^{\circ}$, with an angle difference between the GRIN lens and the homogeneous index lens $<45^{\circ}$ in $82.8 \%$ of the lenses and $<15^{\circ}$ in $45.6 \%$ of the lenses.

Figure 5 shows the age-dependency of the relative astigmatic angle between the lens surfaces. The angle between the anterior and posterior surface astigmatic axes tended to increase with age, but the correlation did not reach statistical significance (slope $=-0.293, r=0.173, P=0.321$ ).

The meridional change of $p 2$ did not show any age dependency ( $r=0.007, P=0.96$, not shown).

\section{Discussion}

In this study, we investigated the contributions of lens surface astigmatism to astigmatism in the lens (in magnitude and axis, for GRIN lenses and lenses with an equivalent refractive index), and potential age dependencies.

We found a significant decrease of the astigmatic magnitude of the anterior surface with age, from positive values toward zero, while the posterior surface astigmatism did not change significantly with age. The lens astigmatism magnitude also had a tendency to decrease. The anterior surface astigmatism correlated well with the lens astigmatism. This observation is based on the fact that the axis of the lens astigmatism is close to the astigmatism axis of the anterior lens surface magnitude. 


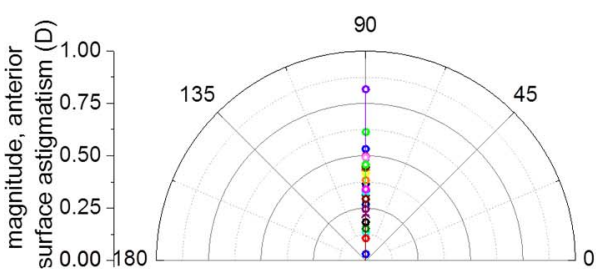

c GRIN lens

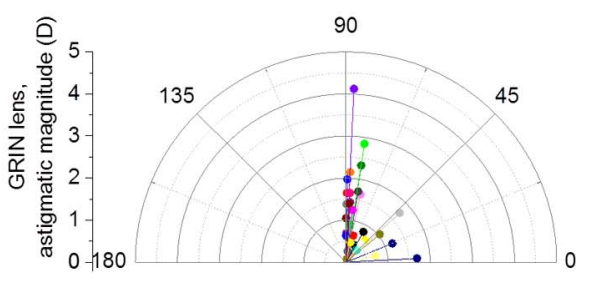

b
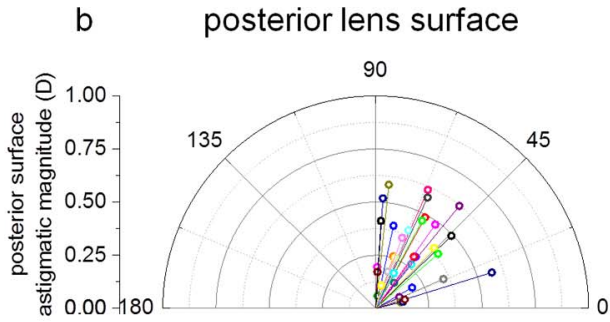

d lens with homogeneous refractive index

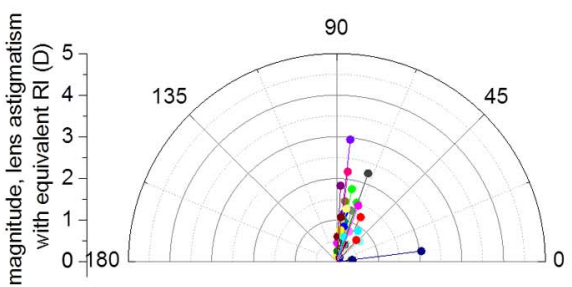

FigURE 4. Power vector plot of (a) anterior surface (the astigmatic axis is vertically aligned), (b) posterior surface, (c) GRIN lens, and (d) lens with homogeneous refractive index. All angles are shown relative to the anterior surface.

In this study, the results ex vivo lack the absolute reference of astigmatic angle with respect to true anatomic features. Despite this, we provided a direct account of the relative axis of astigmatism in the anterior and posterior lens surfaces for the GRIN lens and the lens with homogeneous refractive index. We found that in most cases, the average astigmatic angle between anterior and posterior lens surfaces was $<45^{\circ}$, and that the axis of the GRIN lens was, on average, aligned with the axis of the anterior lens surface.

The magnitude and axis of the internal crystalline lens astigmatism has been a matter of controversy. Traditionally, Javal's rule is assumed, implying a linear relationship between corneal and refractive astigmatism, with a constant offset of 0.5 $\mathrm{D}$ of against-the-rule astigmatism (arising from internal astigmatism). Reports of the magnitude of corneal and refractive astigmatism differ across studies, with some works reporting no change of either, while others report significant changes of one or both. ${ }^{15,39-41}$ In those studies, internal astigmatism is computed indirectly from comparisons of corneal and ocular astigmatism, sometimes from different datasets, which may pose uncertainties.

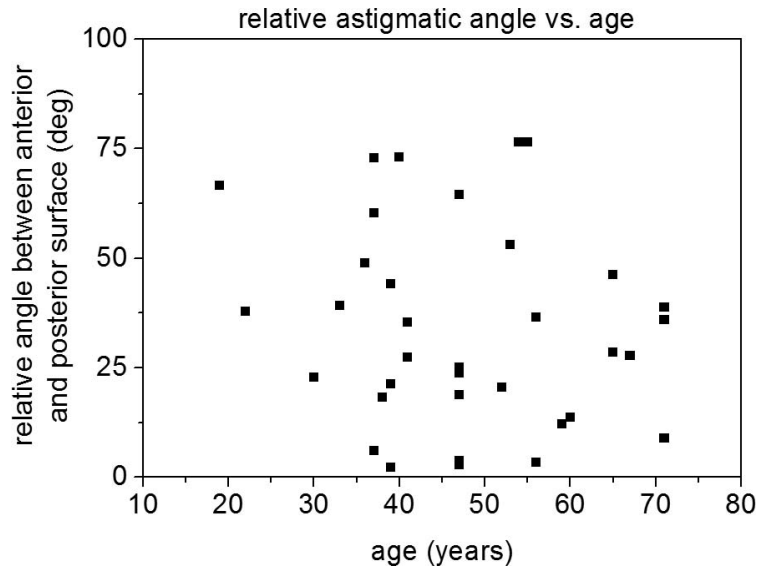

Figure 5. Relative angle between anterior and posterior astigmatic axis with age. The correlation did not reach statistical significance $(r=$ $0.173, P=0.321$ ).
The lack of knowledge of the real orientation to the cornea sets obvious limitations on our study, and on ex vivo lens studies in general. However, direct access on the crystalline lens shape and the reconstruction of GRIN in 3D has allowed us to understand the role of every component in the crystalline lens on its optical properties, astigmatism in particular.

Our results have significant implications for studies quantifying the crystalline lens shape in vivo. In an earlier publication, we showed the possibility of obtaining crystalline lens topography in vivo on three young eyes (ages 28-33), assuming a constant refractive index. The axes of anterior and posterior lens astigmatism were orthogonal in all eyes. ${ }^{51}$ In a similar age group (ages 19-36), we have found on average a smaller angle between anterior and posterior astigmatism $\left(44^{\circ} \pm 18.3^{\circ}\right)$.

The relative small impact of GRIN on astigmatism we found in this study probably arises from the fact that astigmatism is driven by the relative difference in power across meridians, and the meridional component of GRIN is relative small. The results of the study imply that, at least for astigmatism, assuming a constant refractive index would not pose large errors in the reconstruction of the astigmatism of the posterior lens surface measured in vivo.

\section{Acknowledgments}

Supported by Spanish Government Grant FIS2011-25637 (SM), Consejo Superior de Investigaciones Científicas Junta de Ampliacion de Estudios (CSIC JAE)-Pre Program (JB), CSIC i-LINK+ 2012, iLINK +0609 (SM). The research leading to these results has received funding from the European Research Council under the European Union's Seventh Framework Programme (FP7/2007-2013)/ERC Grant Agreement 294099 (SM).

Disclosure: J. Birkenfeld, None; A. de Castro, None; S. Marcos, P

\section{References}

1. Hemenger RP, Garner LF, Ooi, CS. Change with age of the refractive index gradient of the human ocular lens. Invest Ophthalmol Vis Sci. 1995;36:703-707.

2. Garner LF, Smith G. Changes in equivalent and gradient refractive index of the crystalline lens with accommodation. Optom Vis Sci. 1997;74:114-119. 
3. Atchison DA, Smith G. Continuous gradient index and shell models of the human lens. Vision Res. 1995;35:2529-2538.

4. Moffat BA, Atchison DA, Pope JM. Age-related changes in refractive index distribution and power of the human lens as measured by magnetic resonance micro-imaging in vitro. Vision Res. 2002;42:1683-1693.

5. de Castro A, Ortiz S, Gambra E, Siedlecki D, Marcos S. Threedimensional reconstruction of the crystalline lens gradient index distribution from OCT imaging. Opt Express. 2010;18: 21905-21917.

6. Birkenfeld J, de Castro A, Ortiz, S, Pascual, D, Marcos, S. Contribution of the gradient refractive index and shape to the crystalline lens spherical aberration and astigmatism. Vision Res. 2013;86:27-34.

7. Lowe RF, Clark BA. Radius of curvature of the anterior lens surface. Correlations in normal eyes and in eyes involved with primary angle-closure glaucoma. Br J Ophthalmol. 1973;57: 471-474.

8. Brown N. The change in lens curvature with age. Exp Eye Res. 1974; 19:175-183.

9. Glasser A, Campbell MC. Presbyopia and the optical changes in the human crystalline lens with age. Vision Res. 1998;38: 209-229.

10. Glasser A, Campbell MC. Biometric, optical and physical changes in the isolated human crystalline lens with age in relation to presbyopia. Vision Res. 1999;39:1991-2015.

11. Dubbelman M, van der Heijde RGL. The shape of the aging human lens: curvature, equivalent refractive index and the lens paradox. Vision Res. 2001;41:1867-1877.

12. Koretz JF, Cook CA, Kaufman PL. Aging of the human lens: changes in lens shape at zero-diopter accommodation. $J$ Opt Soc Am A. 2001;18:265-272.

13. McLellan JS, Marcos S, Burns SA. Age-related changes in monochromatic wave aberrations of the human eye. Invest Ophthalmol Vis Sci. 2001;42:1390-1395.

14. Amano SAY, Yamagami S, Miyai T, Miyata K, Samejima T, Oshika T. Age-related changes in corneal and ocular higherorder wavefront aberrations. Am J Ophthalmol. 2004;137: 988-992.

15. Artal P, Berrio E, Guirao A, Piers P. Contribution of the cornea and internal surfaces to the change of ocular aberrations with age. J Opt Soc Am A. 2002;19:137-143.

16. Barbero S, Marcos S, Merayo-Lloves J. Corneal and total optical aberrations in a unilateral aphakic patient. J Cataract Refract Surg. 2002;28:1594-1600.

17. Dubbelman M, van der Heijde RGL, Weeber HA. The thickness of the aging human lens obtained from corrected Scheimpflug images. Optom Vis Sci. 2001;78:411-416.

18. Atchison DA, Markwell EL, Kasthurirangan S, Pope JM, Smith G, Swann PG. Age-related changes in optical and biometric characteristics of emmetropic eyes. J Vis. 2008;8(4):29.1-20.

19. Borja D, Manns F, Ho A, et al. Optical power of the isolated human crystalline lens. Invest Ophthalmol Vis Sci. 2008;49: 2541-2548.

20. Moffat BA, Atchison DA, Pope JM. Explanation of the lens paradox. Optom Vis Sci. 2002;79:148-150.

21. Pierscionek BK. Refractive index contours in the human lens. Exp Eye Res. 1997;64:887-893.

22. Jones CE, Atchison DA, Meder R, Pope JM. Refractive index distribution and optical properties of the isolated human lens measured using magnetic resonance imaging (MRI). Vision Res. 2005;45:2352-2366.

23. Kasthurirangan S, Markwell EL, Atchison DA, Pope JM. In vivo study of changes in refractive index distribution in the human crystalline lens with age and accommodation. Invest Ophthalmol Vis Sci. 2008;49:2531-2540.
24. de Castro A, Siedlecki D, Borja D, et al. Age-dependent variation of the gradient index profile in human crystalline lenses. J Mod Opt. 2011;58:1781-1787.

25. de Castro A, Birkenfeld J, Maceo B, et al. Influence of shape and gradient refractive index in the accommodative changes of spherical aberration in nonhuman primate crystalline lenses. Invest Ophthalmol Vis Sci. 2013;54:6197-6207.

26. Birkenfeld J, de Castro A, Marcos S. Contribution of shape and gradient refractive index to the spherical aberration of isolated human lenses. Invest Ophthalmol Vis Sci. 2014;55:25992607.

27. Wong K-H, Koopmans SA, Terwee T, Kooijman AC. Changes in spherical aberration after lens refilling with a silicone oil. Invest Ophthalmol Vis Sci. 2007;48:1261-1267.

28. Heilman BM, Manns F, de Castro A, et al. Changes in monkey crystalline lens spherical aberration during simulated accommodation in a lens stretcher. Invest Ophthalmol Vis Sci. 2015; 56:1743-1750.

29. Borja D, Siedlecki D, de Castro A, et al. Distortions of the posterior surface in optical coherence tomography images of the isolated crystalline lens: effect of the lens index gradient. Biomed Opt Express. 2010;1:1331-1340.

30. Siedlecki D, de Castro A, Ortiz S, Borja D, Manns F, Marcos S. Estimation of contribution of gradient index structure to the amount of posterior surface optical distortion for in-vitro human crystalline lenses imaged by Optical Coherence Tomography. Presented at the 5th European Meeting on Visual and Physiological Optics (EMVPO 2010), Stockholm, Sweden, August 22-24, 2010.

31. Dubbelman M, Sicam VA, van der Heijde GL. The shape of the anterior and posterior surface of the aging human cornea. Vision Res. 2006;46:993-1001.

32. Dunne M, Royston JM, Barnes DA. Normal variations of the posterior corneal surface. Acta Ophthalmol. 1992;70:255261.

33. Oshika T, Tomidokoro A, Tsuji H. Regular and irregular refractive powers of the front and back surfaces of the cornea. Exp Eye Res. 1998;67:443-447.

34. Prisant O, Hoang-Xuan T, Proano C, Hernandez E, Awad S, Azar DT. Vector summation of anterior and posterior corneal topographical astigmatism. J Cataract Refract Surg. 2002;28: 1636-1643.

35. Portello JK, Rosenfield M, O'Dwyer, M. Javal's rule and age. Optom Vis Sci. 1996;73:214.

36. Read SA, Collins MJ, Carney LG. A review of astigmatism and its possible genesis. Clin Exp Optom. 2007;90:5-19.

37. Sicam VA, Dubbelman M, van der Heijde RG. Spherical aberration of the anterior and posterior surfaces of the human cornea. J Opt Soc Am A. 2006;23:544-549.

38. Baldwin WR, Mills D. A longitudinal study of corneal astigmatism and total astigmatism. Am J Optom Physiol Opt. 1981;58:206-211.

39. Keller PR, Collins, MJ, van Saarloost, PP. The relation between corneal and total astismatism. Optom Vis Sci. 1996;73:86-91.

40. Keller PR, McGhee CN, Weed KH. Fourier analysis of corneal topography data after photorefractive keratectomy. J Cataract Refract Surg. 1998;24:1447-1455.

41. Kelly JE, Mihashi T, Howland HC. Compensation of corneal horizontal/vertical astigmatism, lateral coma, and spherical aberration by internal optics of the eye. J Vis. 2004;4(4):262271.

42. Elawad ME. Measurement of Ocular Component Contributions to Residual Astigmatism in Adult Human Eyes [PhD thesis]. Birmingham, UK: Aston University; 1995.

43. Dunne M, Elawad ME, Barnes DA. Measurement of astigmatism arising from the internal ocular surfaces. Acta Ophthalmol Scand. 1996;74:14-20. 
44. Manns F, Fernandez V, Zipper S, et al. Radius of curvature and asphericity of the anterior and posterior surface of human cadaver crystalline lenses. Exp Eye Res. 2004;78:39-51.

45. Sun M, Birkenfeld J, de Castro A, Ortiz S, Marcos S. OCT 3-D surface topography of isolated human crystalline lenses. Biomed Opt Express. 2014;5:3547-3561.

46. Grulkowski I, Gora M, Szkulmowski M, et al. Anterior segment imaging with Spectral OCT system using a high-speed CMOS camera. Opt Express. 2009;17:4842-4858.

47. Rosen AM, Denham DB, Fernandez V, et al. In vitro dimensions and curvatures of human lenses. Vision Res. 2006;46:10021009.
48. Thibos LN, Horner D. Power vector analysis of the optical outcome of refractive surgery. J Cataract Refract Surg. 2001; 27:80-85.

49. Salmon TO, Thibos LN. Videokeratoscope-line-of-sight misalignment and its effect on measurements of corneal and internal ocular aberrations. J Opt Soc Am A. 2002;19:657-669.

50. Maceo BM, Manns F, Borja D, et al. Contribution of the crystalline lens gradient refractive index to the accommodation amplitude in non-human primates: in vitro studies. $J$ Vis. 2011;11(13):23.

51. Ortiz S. Pérez-Merino P, Gambra E, de Castro A, Marcos S. In vivo human crystalline lens topography. Biomed Opt Express. 2012;3:2471-2488. 